\title{
Progress in Refrigeration to Ultralow Temperatures
}

\author{
F. Pobell, Institut für Festkörperforschung, \\ Kernforschungsanlage Jülich
}

Although in terms of degrees, temperatures extremely close to absolute zero were achieved many years ago,

the struggle to attain ever lower temperatures continues.

Even rather spectacular achievements in physics are usually modest in comparison with what is realized in Nature: the most powerful accelerators cannot accelerate particles to the energies of cosmic rays, and it has not yet been possible to generate in a laboratory larger fields, better vacua, larger pressures or higher temperatures than those which exist already in Nature. On the other hand, low temperature physics has far surpassed Nature. The lowest temperature in Nature is limited to $3 \mathrm{~K}$ by the cosmic background radiation. But the lowest temperature to which matter has been refrigerated in the laboratory is $0.000038 \mathrm{~K}$, almost five orders of magnitude lower than Nature's record. The achievements of refrigeration have uncovered phenomena which do not occur anywhere else in the Universe (unless extraterrestrial intelligence exists and is performing experiments at ultralow temperatures). This article describes the recent progress of refrigeration into the microkelvin range by adiabatic nuclear demagnetization.

\section{Development of Refrigeration}

The historical development of refrigeration is shown in Fig. 1. Faraday started to liquefy substances which are volatile at ambient temperature and reached $163 \mathrm{~K}$ in 1845. In 1877, Cailletet as well as Pictet succeeded in producing some fog by compressing and expanding pre-cooled air, reaching temperatures somewhat above 100 $K$. The next steps were the liquefaction and solidification of $\mathrm{O}_{2}$ and $\mathrm{N}_{2}$ by Olszewski and v. Wroblewski (1883; $48 \mathrm{~K})$, and of $\mathrm{H}_{2}$ by Dewar (1898; $12 \mathrm{~K})$.

Today's low-temperature physicists consider Heike Kamerlingh-Onnes as the founder of their field. In 1908, he succeeded in liquefying ${ }^{4} \mathrm{He}$ at $4.2 \mathrm{~K}$ and he discovered superconductivity of metals in 1911. By 1926, Kammerlingh-Onnes had reduced the minimum temperature for his experiments to $0.7 \mathrm{~K}$ by pumping on a liquid helium bath with an enormous battery of pumps; for the first time mankind had reached a temperature below the minimum temperature in Nature.

In the same year, Debye and Giauque suggested a new cooling method - the adiabatic demagnetization of paramagnetic salts - and about $0.25 \mathrm{~K}$ was reached by this method in 1933. The next step, 0.085 $\mathrm{K}$ followed in 1934, and eventually $2 \mathrm{mK}$ was reached in 1953. Similar lower limits exist for two other cooling techniques developed since then, the ${ }^{3} \mathrm{He}-{ }^{4} \mathrm{He}$ dilution refrigeration ( $T \cong 2 \mathrm{mK}$ ), and the cooling by solidification of liquid ${ }^{3} \mathrm{He}$ (Pomeranchuk-cooling; $T \cong 1 \mathrm{mK}$ ). The only known method for refrigeration into the submillikelvin range is an "advanced" version of the magnetic cooling: "adiabatic demagnetization of nuclear magnetic moments", suggested already in 1934 by Gorter as well as by Kurti and Simon.

The limit for cooling by adiabatic demagnetization of paramagnetic salts is the spontaneous magnetic ordering of the electronic magnetic moments when their interaction wins against the thermal disorder. Because nuclear moments are about three orders of magnitude weaker, their ordering usually occurs at substantially lower temperatures. The possibility of cooling to these lower temperatures unfortunately entrains many substantial experimental problems. These are: first, the high starting fields of the order of $10 \mathrm{~T}$ and low starting temperatures of the order of $10 \mathrm{mK}$, necessary to polarize the weak nuclear moments partially and to obtain a sufficient initial entropy reduction; second, the small cooling capacity of nuclear magnetic refrigerators which can only withstand heat leaks of the order of nanowatts. And last but not least, the long relaxation times which are often several hours or even days make experiments very difficult at ultralow temperatures.

The first nuclear cooling experiment, performed in 1956 by Kurti et al. made the experimental problems obvious. Starting from $3 \mathrm{~T}$ and $12 \mathrm{mK}$, they could cool $\mathrm{Cu}$ nuclei to about $1 \mu \mathrm{K}$ but the $\mathrm{Cu}$ electrons and the lattice stayed at $12 \mathrm{mK}$, and the nuclei warmed up to the starting temperature within a few minutes. The problem in nuclear refrigeration is not the cooling of nuclei but the transfer of the ultralow temperature from the nuclei to the electrons and lattice of the refrigerant, and from there to an experimental sample and to thermometers, and eventually to maintain it while performing the experiment.

\section{Nuclear Adiabatic Demagnetization Refrigeration}

Fig. $2 \mathrm{a}$ is a schematic representation of a modern single stage nuclear refrigerator as they have been developed particularly by Lounasmaa's group in Helsinki. The nuclear stage consists of 10 to 40 moles of copper in the form of insulated thin wires (to keep eddy current heating small during demagnetization). The lower part of the wires is located in a superconducting solenoid. The wires are long enough to

Fig. 1 - Historical development of the minimum temperature to which samples have been refrigerated by liquefaction of the indicated gases ( $\mathbf{\Delta}$ ) or by adiabatic demagnetization of electronic magnetic moments (-) and of nuclear magnetic moments ( $\mathbf{n}$ ), respectively. The lowest temperature to which a sample has been refrigerated is $38 \mu \mathrm{K}(\bullet)$, reached in 1980 in Jülich.

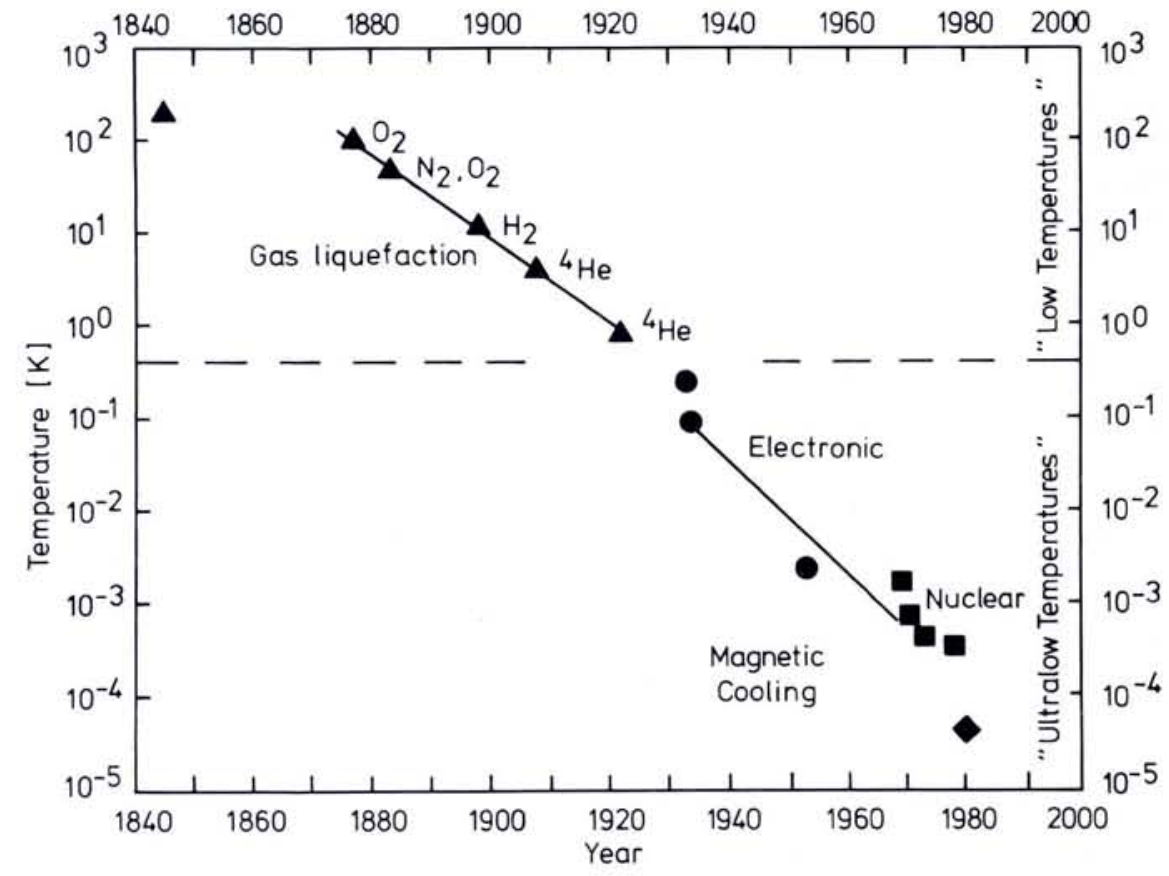




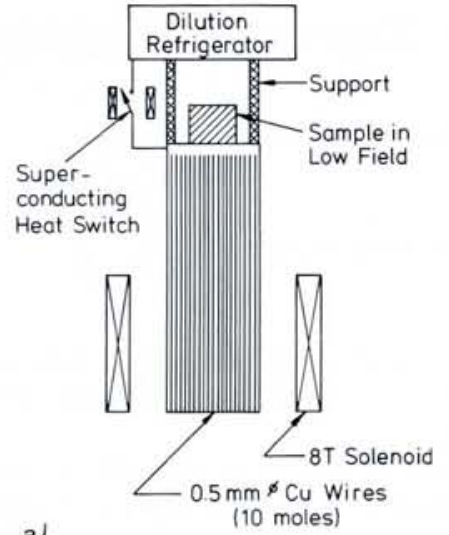

a)

Single-Stage Nuclear Demagnetization Refrigerator

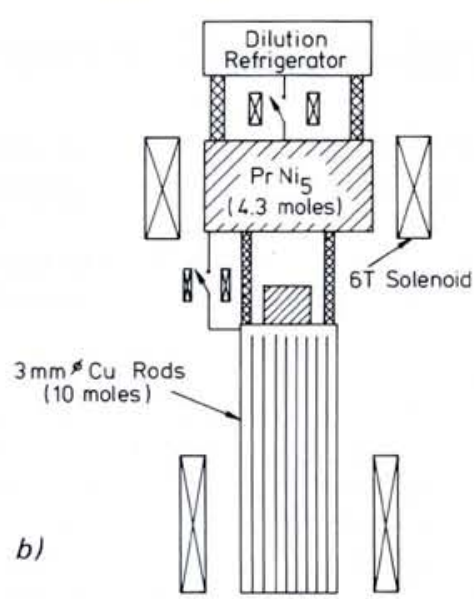

Double-Stage Nuclear Demagnetization Refrigerator

Fig. 2 - Schematic of a single-stage Cu nuclear refrigerator and of the Jülich double-stage $\mathrm{PrNi}_{5} \mathrm{Cu}$ nuclear refrigerator. The extra $\mathrm{PrNi}_{5}$ stage decreases the starting temperature for demagnetizing the $\mathrm{Cu}$ in the double-stage design. It also decreases the heat leak transmitted to experiments, thermometers and the Cu nuclear stage.

cool a sample located outside the high field region. After the solenoid has been energized to a typical value of $8 \mathrm{~T}$, the copper is pre-cooled by a dilution refrigerator to about $15 \mathrm{mK}$. These rather demanding starting fields and temperatures have only been made accessible by the development of superconducting magnets and ${ }^{3} \mathrm{He}-{ }^{4} \mathrm{He}$ dilution refrigerators in the last two decades. The limits set by the technology of these two tools determine the initial reduction in the nuclear entropy of the $\mathrm{Cu}$ which usually is only about 3-5\%. This entropy reduction determines the cooling capacity of the refrigerator.

The thermal contact from the nuclear refrigerator to the dilution refrigerator is via a heat switch which consists of a superconducting metal. In the superconducting state, the thermal conductivity of this switch is very low; but if the metal is driven into the normal state by a small magnetic field, its thermal conductivity becomes as high as that of any normal metal. When the pre-cooling by the dilution refrigerator is complete, the heat switch is made superconducting to isolate thermally the copper nuclear refrigeration stage; the demagnetization is then begun. It continues slowly to reduce eddy current heating, and to keep thermal equilibrium between nuclei and electrons. If the demagnetization is performed adiabatically, without losses, the entropy of the $\mathrm{Cu}$ nuclei remains constant and the temperature decreases proportional to the field.

About twenty such nuclear refrigerators have been built between 1970 and 1980 . Some can cool samples to about $0.5 \mathrm{mK}$ and the best to $0.2 \mathrm{mK}$. A great number of exciting experiments in this new temperature range have been performed, particu larly to investigate the superfluidity of liquid ${ }^{3} \mathrm{He}$ below $2.7 \mathrm{mK}$ (detected in 1972) and the nuclear antiferromagnetism of solid ${ }^{3} \mathrm{He}$ below $1 \mathrm{mK}$ (detected in 1974). Too

large heat leaks and too small original entropy reductions prevent the refrigerators from doing better. But there are important experiments which require lower temperatures, demanding new concepts in refrigerator design. Some of these have been realized in the Jülich double-stage refrigerator which demonstrates the feasibility of doing solid state physics down to $40 \mu \mathrm{K}$. In addition, a nuclear refrigerator has been built in Helsinki particularly for the polarization of nuclear spins and has reached a nuclear spin temperature of about $50 \mathrm{nK}$.

\section{Jülich Double-Stage Nuclear Refrigera-} tor

The cooling capacity of a nuclear refrigerator can be increased by increasing the original entropy reduction. This requires the temperature to be lowered or the magnetic field to be increased at the start of the demagnetization. A substantial increase of the magnetic field above the usual $8 \mathrm{~T}$ attainable with a superconducting magnet is difficult and expensive. The rate $\dot{S}_{D R}$ at which a typical dilution refrigerator can remove entropy, as shown in Fig. 3 , becomes rather small below $15 \mathrm{mK}$ and the refrigeration to $5 \mathrm{mK}$ of a few moles of copper in a field of $8 \mathrm{~T}$ for example, with a dilution refrigerator usually requires a prohibitively long time making other approaches necessary.

The improvements depend largely on having two nuclear demagnetization stages which operate in cascade. The first has to pre-cool the second stage, which is subsequently demagnetized to the ultralow final temperature. The first stage is distinguished by doing all its work at relatively "high" temperatures, above $5 \mathrm{mK}$, but here it must absorb the very large nuclear magnetic heat of the second, magnetized nuclear stage. Materials which can meet this requirement are metallic Van Vleck parama- gnets. In these materials, a large hyperfine field is induced at the nuclei by polarization of the $4 \mathrm{f}$ electrons through the application of an external field, resulting in a large nuclear polarization and entropy reduction. One of the best Van Vleck paramagnets for nuclear demagnetization is the compound $\mathrm{PrNi}_{5}$, as was first shown by Andres in 1977. In this compound, the ${ }^{141} \mathrm{Pr}$ nuclei see a field which is about a factor of twelve larger than the externally applied field. Fig. 3 includes entropy curves for $\mathrm{PrNi}_{5}$ from which it will be seen that the nuclei can be highly polarized and most of the nuclear entropy removed over the range of temperatures in which a dilution refrigerator is still very effective.

$\mathrm{PrNi}_{5}$ is an excellent nuclear refrigerant but it cannot produce temperatures below about $0.2 \mathrm{mK}$ because spontaneous nuclear ferromagnetic ordering of the $\mathrm{Pr}$ nuclei occurs at $0.4 \mathrm{mK}$ (see Fig. 3). Spontaneous nuclear ordering is also expected in $\mathrm{Cu}$ but only at a temperature near $10^{-7} \mathrm{~K}$ because no large hyperfine field acts on the $\mathrm{Cu}$ nuclei. To take full advantage of the possibilities of a double-stage design one has thus to use a hyperfine enhanced material with a large nuclear cooling capacity in the low-millikelvin range in the first nuclear stage, to pre-cool a second stage consisting of a material with a very low nuclear magnetic ordering temperature. Both subs tances should have a negligible nuclear electric quadrupole interaction and they have to be metallic to be able to transport heat at ultralow temperatures.

The manner in which a $\mathrm{PrNi}_{5}$ stage is added to the system is depicted schematically in Fig. 2b. Besides the extra refrigerant $\mathrm{PrNi}_{5}$, another superconducting magnet and another heat switch are required. During pre-cooling, both heat switches are in the high-thermal conductivity, normal state and both solenoids are generating their full field. About $70 \%$ of the entropy of the $\mathrm{PrNi}_{5}$ in a field of $6 \mathrm{~T}$ is already removed at $25 \mathrm{mK}$ where the dilution refrigerator is still quite powerful. At about $25 \mathrm{mK}$, the stages are then thermally isolated from the dilution refrigerator and the $\mathrm{PrNi}_{5}$ is demagnetized at the end of which the copper temperature is 4 to $5 \mathrm{mK}$. At the latter temperature, $27 \%$ of the nuclear entropy of copper is removed, to be compared with $4 \%$ if the copper is cooled to only $15 \mathrm{mK}$. This greatly increases the amount of heat the copper can absorb when it is demagnetized. For this last step, the lower heat switch is opened and the $\mathrm{Cu}$ is demagnetized from 8 $\mathrm{T}$ to $0.01 \mathrm{~T}$ exponentially with time (see Fig. 4).

The performance of the refrigerator is shown in Fig. 4 which presents the temperatures measured at the location of the experiments about $20 \mathrm{~cm}$ above the centre of the demagnetized $\mathrm{Cu}$ as a function of time and of demagnetizing field. This figure also demonstrates the very long relaxation times typical for ultralow temperatures. 


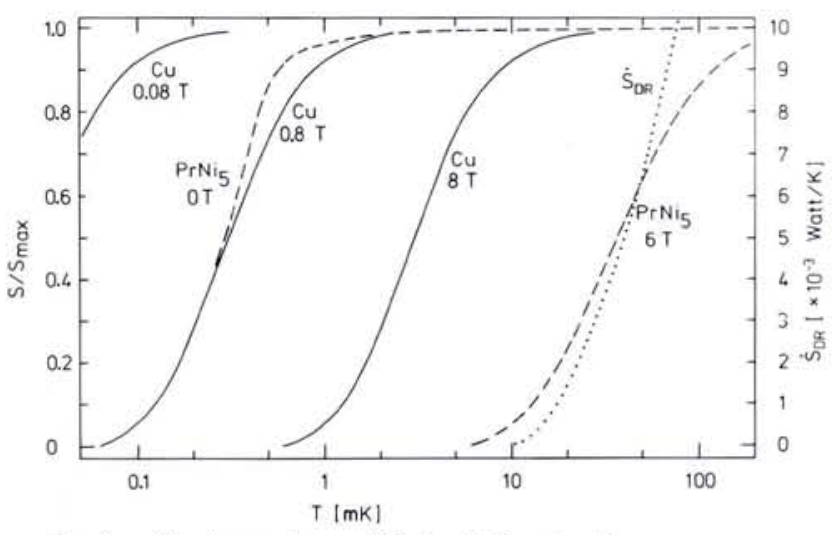

Fig. 3 - Nuclear entropy of Cu (solid lines) and of $\mathrm{PrNi}_{5}$ (dashed lines) in various fields, compared to the rate of entropy reduction $S_{D R}$ of a typical ${ }^{3} \mathrm{He}-{ }^{4} \mathrm{He}$ dilution refrigerator idotted line). For $\mathrm{Cu}, S_{\max }=11.5 \mathrm{~J} / \mathrm{K}$ mole and for $\mathrm{PrNi}_{5}, S_{\max }=14.9 \mathrm{~J} / \mathrm{K}$ mole. Owing to the hyperfine enhancement of the external field, the reduction of nuclear entropy of $\mathrm{PrNi}_{5}$ occurs at temperatures at which $S_{D R}$ is still large.

Only four days after the demagnetization was completed, did the thermometer indicate that the experimental samples had relaxed to about $40 \mu \mathrm{K}$. The samples can stay below $50 \mu \mathrm{K}$ for ten days giving ample time for experiments. The nuclei in the centre of the demagnetized copper are at $5 \mu \mathrm{K}$, and its electrons are only about $2 \mu \mathrm{K}$ "hotter". The sample at the upper end of the $\mathrm{Cu}$ stage is so much "hotter" than the centre of the Cu stage because of a heat leak which totals nevertheless between 0.1 and $1 \mathrm{nW}$ only.

Once a new temperature regime is entered, thermometry is, of course, as important as refrigeration. The thermometric method most applied in the microkelvin range is nuclear magnetic resonance. At present, there exists no established temperature scale in the milli- and microkelvin range. The temperatures derived are believed to be accurate to about $\pm 5 \%$.

\section{Heat Leaks Limiting Minimum Tempe- ratures}

The performance of today's nuclear refrigerators, their minimum temperature and the time they can stay below a certain temperature, are limited by heat leaks and the finite thermal conductivity of the materials used. One of the main achievements in ultralow temperature technology has therefore been the reduction by careful design of heat leaks of obvious origin, such as conduction through mechanical connections like supports, heat switches or electrical leads, by residual gases, by radiation, or an input from RF sources - to a level below $10^{-10}$ or $10^{-11} \mathrm{~W}$. But there are other heat sources which are less familiar and often much harder to control. One example is the heat generated by vibration. A careful consideration of these and the very rigid construction adopted have, no doubt, contributed to the success of our refrigerator. Heat generated by ionization caused by the warm-up.

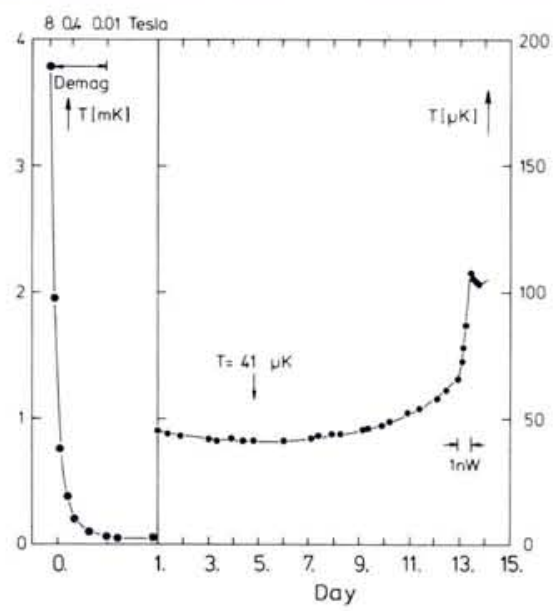

Fig. 4 - The left part shows the decrease of temperature, starting at $3.8 \mathrm{mK}$, of a sample in the Jülich double-stage nuclear refrigerator when the magnetic field acting on the Cu refrigerant is decreased exponentially in time from $8 \mathrm{~T}$ to $0.01 \mathrm{~T}$. The right part shows, in an expanded temperature scale $(\mu K)$ and a compressed time scale, the development of the sample temperature after demagnetization; it takes about 4 days until it has relaxed to the minimum temperature. The sample was kept below $50 \mu \mathrm{K}$ for ten days. On the 13th day after demagnetization, $1 \mathrm{nW}$ was supplied to accelerate

cosmic rays passing through the nuclear stage, or the experimental region of a nuclear refrigerator, can also be of the order of $10^{-10}-10^{-11} \mathrm{~W}$.

Nevertheless, the performance of most ultralow temperature refrigerators, including our own, is mainly limited by the unexpected observation that the main part of the measured heat leak is time dependent with relaxation times of the order of one week. We have therefore, to keep the refrigerator at low (not at ultralow) temperatures for a month or two until this part of the heat leak has decayed so it becomes comparable to the above mentioned timeindependent contributions. After each warm-up to room temperature, the heat leak at low temperatures starts again at the same value of about $30 \mathrm{nW}$. The timedependent contribution obviously must evolve in the low temperature part of the refrigerator itself.

Possible mechanisms for this very slow energy release are, for example, tunnelling transitions of protons in non-crystalline substances or the relaxation of mechanical strain. All connections between different materials require glueing, soldering, alloying, squeezing, or welding. They therefore, almost unavoidably, introduce one or more potential internal heat sources. A detailed investigation of the physics of these heat storage and release mechanisms is an indispensable requirement if further progress in refrigeration is the aim. Besides this technological aspect, the physics of these mechanisms is of great interest.

\section{Conclusion}

Low temperature physics has entered a new and exciting stage on the road to absolute zero by making the microkelvin range accessible, and there are a great number of interesting and important problems to solve. Fundamental discoveries have often been the outgrowth of techno- logical developments: the most recent examples in low-temperature physics are the discoveries of superfluidity of liquid ${ }^{3} \mathrm{He}$ and of nuclear anti-ferromagnetism of solid ${ }^{3} \mathrm{He}$ in the low millikelvin range. The present technology seems to be limited to the temperature range above about $10 \mu \mathrm{K}$, mostly because of heat leaks - already minute - , the finite thermal conductivity of the purest metals and the excessively long time constants. If we attempt to reach lattice temperatures below $1 \mu \mathrm{K}$, new ideas and changes in refrigerator design seem to be necessary.

The motivation for entering any new regime like a new temperature range, is the fact that nobody can predict which new, unknown phenomena may be hidden in this range. The third law of thermodynamics tells us that we shall never reach absolute zero. But each new decade in temperature may contain as many exciting phenomena as the foregoing, and there are an infinite number of decades between the explored temperature ranges - the most recent are just starting to become explored - and absolute zero.

\section{BIBLIOGRAPHY}

A description of the historical development of low temperature physics can be found in: Mendelssohn K., The Quest for Absolute Zero (McGraw Hill) 1966.

A description of modern refrigeration and physics at ultralow temperatures can be found in: Lounasmaa O.V., Experimental Principles and Methods below 1 K (Academic Press, London) 1974; Betts D.S., Refrigeration and Thermometry below $1 \mathrm{~K}$ (Sussex University Press) 1976.

The design and performance of the Jülich Double-Stage Nuclear Refrigeration is discussed in: Müller R.M., Buchal Ch., Folle H.R., Kubota M. and Pobell F., A Double-Stage Nuclear Demagnetization Refrigerator, Cryogenic 20 (1980) 395; Pobell F., "The Quest for Ultralow Temperatures: What are the Limitations?", Physica $B$ (1982) 